Abstract S33 Table 1 Spearman's correlation for sputum colour at all exacerbations

\begin{tabular}{lll}
\hline & \multicolumn{2}{l}{ Sputum Colour } \\
\cline { 2 - 3 } & rho & p value \\
\hline FEV1\% & 0.26 & $<0.01$ \\
Sputum & & \\
Neutrophils (count) & 0.66 & $<0.01$ \\
Neutrophils (\%) & 0.62 & $<0.01$ \\
Macrophages (count) & -0.38 & $<0.01$ \\
Macrophages (\%) & -0.48 & $<0.01$ \\
Lymphocytes (count) & -0.22 & $<0.05$ \\
Lymphocytes (\%) & -0.23 & $<0.05$ \\
Blood & & \\
Neutrophils (10*9/L) & 0.20 & $<0.05$ \\
CRP (mg/L) & 0.47 & $<0.01$ \\
Fibrinogen (g/L) & 0.32 & $<0.01$ \\
Procalcitonin (ng/ml) & NS & NS \\
EXACT-PRO & NS & NS \\
CAT & NS & NS \\
\hline
\end{tabular}

FEV1: Forced Expiratory Volume in 1 second; CRP: C Reactive Protein; EXACT-PRO: The Exacerbations of Chronic Pulmonary Disease Tool Patient Reported Outcome; CAT: The COPD Assessment Tool; NS: No significance

Introduction Acute exacerbations of COPD have a major impact on patients' health related quality of life (HRQoL), and the utilisation of health care resources. Current guidelines recommend oral corticosteroids and/or antibiotics for the treatment of acute exacerbations of COPD based on patients' symptoms. With increasing bacterial resistance to antibiotics and the rising costs of COPD treatment, further research into diagnostic tools to aid the management of COPD in its stable and exacerbating states is required. Sputum colour (SC) is an accessible marker of underlying bronchial inflammation. We investigated the contribution of objective measures of SC as a component of the clinical assessment of exacerbations and relationships with symptom severity. Methods Data from 36 patients with moderate to very severe COPD was assessed in this prospective observational cohort study (AERIS). There were 122 exacerbations in total over a year. Sputum and blood sampling were performed at enrolment, routine follow up and exacerbation visits. A five-point sputum colour chart was developed to objectively report the SC. Sputum samples from all visits were graded against this chart by the trained laboratory staff. Data from mild, moderate and severe exacerbations were included in the analysis.

Results We found a correlation between SC at exacerbations and disease severity (FEV1\%) at exacerbations. SC was also related to sputum neutrophilia at exacerbations. SC was significantly associated with systemic markers such as blood neutrophilia, CRP and fibrinogen. Interestingly, we observed no statistically significant correlation between SC and Procalcitonin levels. We also found no statistically significant relationship between SC and symptom scores (CAT and EXACT-PRO) at exacerbations. However, we found a significant association between CAT and EXACT-PRO scores (rho 0.46; $\mathrm{p}<0.01$ ).

Conclusion We observed that visual colour score of sputum at exacerbations is related to underlying airway and systemic inflammation but not to symptom scores. The use of a SC combined with other clinical and laboratory biomarkers, as part of a multicomponent diagnostic tool, may further improve its clinical utility to better guide effective exacerbation treatment. Further analysis of the full AERIS cohort will explore this.
Pulmonary arterial hypertension: scientific advances

\section{S34 BMPR-II DEFICIENCY LEADS TO AN INCREASE IN LUNG EGG DEPOSITION, PULMONARY VASCULAR REMODELLING AND AN ABNORMAL LIVER VASCULATURE IN MICE CHRONICALLY INFECTED WITH S. MANSONI}

A Crosby, E Soon, F Jones, M Southwood, L Haghighat, M Toshner, T Raine, I Horan, P Yang, A Davenport, S Moore, P Wright, D Dunne, N Morrell. Cambridge University, Cambridge, UK

\subsection{6/thoraxjnl-2014-206260.40}

Rationale and objectives Schistosomiasis is the world-wide leading cause of pulmonary arterial hypertension $(\mathrm{PAH})$ and is particularly prevalent in developing countries. More than $80 \%$ of patients with familial PAH in the western-world have a mutation in bone morphogenetic protein type-II receptor (BMPR-II), which is a member of the transforming growth receptor-beta (TGF-b) superfamily and is important in cell proliferation and differentiation. The aim of the study was to determine if mice with a heterozygous null mutation in BMPR-II are more susceptible to pulmonary vascular remodelling induced by S. mansoni infection, compared with wild-type littermates.

Methods Wild-type (BMPR-II+/+) and BMPR-II heterozygous (BMPR-II+/-) C57/BL6 mice were infected percutaneously with S. mansoni. Seventeen weeks post-infection right ventricular systolic pressure (RVSP), right ventricular hypertrophy (RVH), liver and lung egg counts were measured. Pulmonary vascular remodelling and liver histology were assessed by morphometry, following immunohistochemistry. Lung, liver and serum cytokines were also measured. A macrophage phagocytosis assay and in vivo bead assay were also performed.

Measurements and main results At 17 weeks post-infection there was a significant increase in pulmonary vascular remodelling associated with a significant increase in egg deposition and cytokines in the lung, in BMPR-II+/- mice. Furthermore, there was a positive correlation between lung egg deposition and pulmonary vascular wall thickness. Additionally, there was a significant dilatation of the central hepatic vein in the BMPR-II+/infected mice compared with the BMPR-II $+/+$ infected mice. However, no differences in RVSP, RVH or liver egg deposition were found.

Conclusions This study has shown that mice deficient in BMPR-II are more susceptible to pulmonary vascular remodelling induced by $S$. mansoni which is directly correlated to an increase in egg burden in these mice. Additionally, we have shown that BMPR-II+/- mice have an abnormal liver vasculature, which may be responsible for increased egg shunting into the lungs.

\section{S35 BMP9 AND BMP10 MEDIATE CONNEXIN EXPRESSION IN ENDOTHELIAL CELLS: IMPLICATIONS FOR PAH AND HHT}

PD Upton, JM Wilkinson, KA Wiggins, NW Morrell. University of Cambridge, Cambridge, UK

10.1136/thoraxjnl-2014-206260.41

Background Germ-line mutations in the bone morphogenetic protein type-II receptor, BMPR-II, underlie $80 \%$ of heritable 
pulmonary arterial hypertension (PAH) cases and approximately $25 \%$ of idiopathic PAH cases. PAH may arise due to endothelial dysfunction as mice with BMPR-II deficiency exhibit increased pulmonary vascular permeability.

BMP9 is an endothelial quiescence factor and is thought to maintain the integrity of the endothelium. We previously reported that BMPR-II and ALK1 are the key receptors through which BMP9 inhibits the proliferation of human pulmonary artery endothelial cells (hPAECs). We hypothesised that BMPR-II deficiency impacts on endothelial cell connectivity and may contribute to endothelial dysfunction in PAH.

Methods Human pulmonary artery endothelial cells were obtained from Lonza and blood outgrowth endothelial cells (BOECs) were isolated from peripheral blood of unaffected controls or PAH patients with identified BMPR-II mutations. Cells were transfected with siRNAs targeting BMPR-II followed by stimulation with BMP9. RNA was extracted and the expression of candidate genes determined by quantitative PCR. Further siRNA studies were performed for ALK1 and endoglin siRNAs. The promotion of gap junction assembly by BMP9 and BMP10 were assessed by immunofluorescence, Western blotting and functionally using parachute assays.

Results Screening of candidate BMP9-induced junctional and structural proteins highlighted a subset of endothelial connexins that are BMP9 and BMP10-responsive and dependent on BMPR-II and ALK1. BMP9 and BMP10 increased the expression of the connexins, assessed by Western blotting and immunostaining. In addition, BMP9 and BMP10 significantly increased the transfer of calcein from labelled donor cells to unlabelled acceptor cells, indicating a promotion of endothelial cell connectivity.

Conclusion In addition to their roles promoting endothelial quiescence, BMP9 and BMP10 directly promote endothelial cell connectivity by increasing connexin expression and assembly. The central contributions of BMPR-II and ALK1 to this process may implicate impaired endothelial connectivity as a pathological component of PAH and HHT.

\section{S36 FERROPORTIN IS EXPRESSED IN HUMAN PULMONARY ARTERY SMOOTH MUSCLE CELLS: IMPLICATIONS FOR PULMONARY ARTERIAL HYPERTENSION}

L Ramakrishnan, S Mumby, JS Wort, G Quinlan. Imperial College London, London, UK

\subsection{6/thoraxjnl-2014-206260.42}

Background Pulmonary Arterial Hypertension (PAH) is a rare but fatal condition manifested by pulmonary vascular remodelling, increased pulmonary vascular resistance and right-heart failure. Disruption in iron handling and anaemia, caused by elevated iron-regulatory hormone hepcidin, is observed in PAH. Ferroportin the only known cellular iron-export protein is downregulated by hepcidin. As such, iron supplementation as a therapy is currently under clinical trial. However, it is also known that iron is both pro-oxidant and pro-proliferative. Latest evidence also points to sub-clinical haemolysis and the presence of free haemoglobin in PAH patients. We hypothesised that ferroportin would be expressed; be responsive to hepcidin challenge and have implications for the proliferation of human pulmonary artery smooth cells (hPASMCs).

Methods The mRNA levels of ferroportin was measured by RT-PCR, the protein expression was detected by western-blot analysis and quantified by ELISA. The sub-cellular distribution of

\section{Change in ferroportin levels $24 \mathrm{~h}$ after hepcidin treatment in PASMCs}
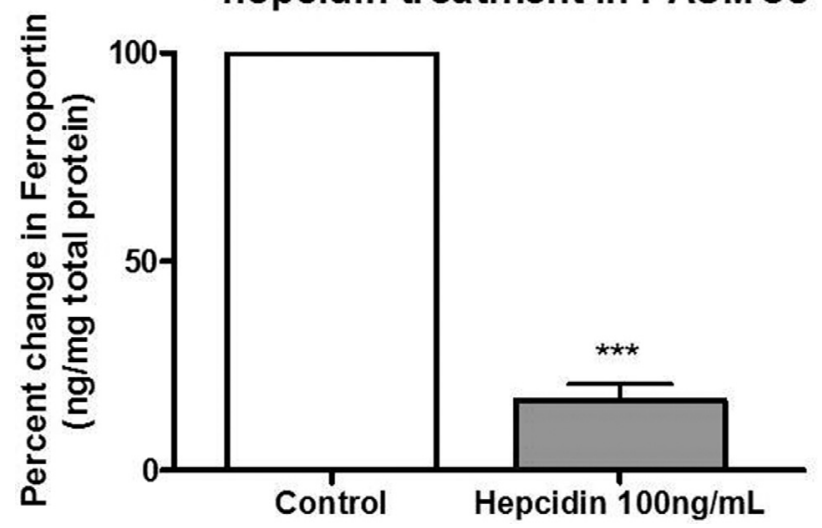

Abstract S36 Figure 1 Confluent PASMCs (passage 4-8) were treated with $100 \mathrm{ng} / \mathrm{ml}$ hepcidin for 22-24 h and total protein extracted using cell-lysis buffer. Ferroportin expression was quantitated using an ELISA kit and normalised to total protein estimated by bradford reagent. $n=4,{ }^{* *}=p<0.001$

ferroportin was visualised by immunocytochemistry (ICC). hPASMCs were pre-incubated with or without free haemoglobin and further challenged with increasing doses of hepcidin and the proliferative responses assessed by cyquant and/or BrdU incorporation assays. Some cells were also pre-incubated with LY2928057 (monoclonal antibody against ferroportin that stabilises cellular expression, Eli-Lily) in proliferation assays.

Results Basal ferroportin mRNA was detected in hPASMCs, but the mRNA levels were largely unaltered with hepcidin exposure $(\mathrm{n}=3)$. A $\sim 50 \mathrm{KDa}$ protein band representing ferroportin was detected under resting conditions while hepcidin challenge caused decrease in ferroportin protein levels (Figure 1). Basal ferroportin was uniformly distributed in the cells; however hepcidin treatment led to intense punctate/vesicular staining $(n=3)$. Finally, exposure to free haemoglobin alone or along with hepcidin increased proliferation of hPASMCs by $13.6 \%$ and $12.4 \%$ ( $p$ $<0.05, \mathrm{n}=3$ ) respectively. Interestingly, pre-incubation of the cells with LY2928057 partly reversed this effect.

Conclusion This is the first report of ferroportin expression and regulation in hPASMCs. We suggest that targeting and manipulating the hepcidin-ferroportin axis using LY2928057 might prove a novel therapeutic approach for PAH.

\section{S37 VASCULAR ENDOTHELIAL CELL GROWTH FACTOR-A (VEGF-A) SIGNALLING AND NEOVASCULARISATION OF PULMONARY ENDARTERECTOMY MATERIAL IN CHRONIC THROMBOEMBOLIC PULMONARY HYPERTENSION (CTEPH)}

M Southwood, C Hadinnapola, E Moseley, D Jenkins, M Goddard, K Sheares, M Toshner, J Pepke-Zaba. Papworth Hospital NHS Foundation Trust, Cambridge, UK

\subsection{6/thoraxjnl-2014-206260.43}

Background Despite recent advances in the medical treatment of patients with CTEPH, relatively little is understood surrounding the underlying pathological mechanisms. Many patients have a historical documented venous thromboembolic event (VTE) and consequently, failed resolution of an acute VTE has been proposed as a key initiating factor in the subsequent development of CTEPH. Here we investigated VEGF-A levels, a key regulator of angiogenesis, in CTEPH patients prior to and following 